\title{
Emergency Control Concepts for Future Power Systems
}

\author{
Stephan Koch* Matthias D. Galus* Spyros Chatzivasileiadis * \\ Göran Andersson * \\ * ETH Zurich, EEH - Power Systems Laboratory \\ Physikstrasse 3, 8092 Zurich, Switzerland. \\ E-Mail: \{koch, galus, spyros, andersson\}@eeh.ee.ethz.ch. \\ Web Site: www.eeh.ee.ethz.ch.
}

\begin{abstract}
In this paper, three approaches to increasing power system security are presented which are particularly relevant with respect to emerging fundamental changes in the operation paradigms of electric grids. Special attention is given to the challenge of maintaining system stability and security of supply in the presence of distributed and fluctuating renewable power generation and the integration of electro-mobility into the distribution grid. The proposed remedies, tailored for integration into future "smart grid" communication and control structures, consist of introducing controlled islanding schemes for interconnected transmission grids, highly distributed under-frequency load shedding mechanisms on the customer level, and controlled disconnection of electric vehicles in case of a distribution grid overloading. The motivation and principles of operation of each measure are presented and illustrated by a simulation example.
\end{abstract}

Keywords:

Electric power systems, system security, transient stability, frequency stability, emergency control, controlled islanding, load shedding, electric vehicles.

\section{INTRODUCTION}

Interconnected electric power systems are currently facing the probably most rapid and fundamental changes since their successive construction and expansion in the course of the $20^{\text {th }}$ century. Liberalized electricity markets have been established in many countries, leading to a utilization of the power system for market-based trading, and consequently to increased interarea power flows, grid congestions, and lower operational security margins (Belmans, 2007). The requirement to decarbonize the energy system has led, and will continue to lead, to largescale deployments of renewable energy conversion systems, which are often decentralized and have fluctuating in-feed characteristics, requiring novel management strategies. In the same way, the expected future integration of large plug-in hybrid electric vehicle fleets is likely to pose problems for system operation if not properly managed (Pecas Lopes et al., 2009).

Maintaining the current high reliability of electric power supply is expected to become increasingly challenging due to the outlined developments. Blackouts and disturbances such as in the US and Italy in 2003, and in central Europe in 2006 (Bialek, 2007) have demonstrated the vulnerability of modern power systems. The analysis of the latter event (UCTE, 2007) has shown that the uncontrolled nature of Distributed Generation disconnection and reconnection (in this case mostly caused by wind turbines without fault-ride-through capability) has made the mitigation of the disturbance more difficult. This underlines the necessity for enhancing the controllability of both generation and load and the application of new system operation strategies in normal operation and emergency situations.

This paper is to be understood as an overview of a selection of recognized threats to security and reliability of the power system. The discussed emergency control strategies can make a contribution to the security and reliability of future power systems by addressing some of the novel challenges. All of them require infrastructure for communication and control on all grid levels, for the second and third case even including the final customer level, as envisioned in the idea of a comprehensive "smart grid" infrastructure (He et al., 2010).

The article is structured as follows: In Section 2, an approach for controlled islanding of parts of the power system is presented which is able to prevent it from progressing into transient instability. The algorithm is based on a $k$-means clustering technique and can minimize load-generation imbalances in the islands. Section 3 presents a customer-level under-frequency load shedding approach, which is capable of achieving a quick load reduction while maintaining the supply of vital loads and keeping generation units in the distribution grid connected. In Section 4, effects of plug-in hybrid electric vehicles on the grid and approaches to avoid the overloading of distribution system infrastructure are discussed. Section 5 draws some conclusions.

\section{CONTROLLED ISLANDING}

\subsection{Motivation}

Controlled islanding has been proposed by a number of researchers as an appropriate corrective control measure against large disturbances. Several schemes have been described in the literature, while some have been tested through practical implementations (Bollen and Samuelsson, 2009) or are in the planning phase for implementation in the near future (Sena et al., 2010). Controlled islanding schemes split the power system into smaller islands. In this way, a disturbance can be contained and prevented from leading to cascading failures. As a result, tripping of the generators due to loss of synchronism is 
avoided. As more generators remain online, less load needs to be shed and faster restoration times can be achieved.

Two main objectives are sought when designing a controlled islanding scheme. The first is the formation of stable islands. The second is the minimum load-generation imbalance within each island, in order to minimize the total amount of load shedding. Li et al. (2005) propose a controlled islanding approach based on graph spectral methods. An approach presented by You et al. (2004) suggests a slow-coherency grouping of the generators and the determination of the minimum cut-sets in order to form the islands. Sun et al. (2005) describe a real-time controlled islanding scheme with an extensive study of the 118bus system based on a graph theoretic approach called OBDD (Ordinary Binary Decision Diagram).

With the increasing penetration of fluctuating power sources and demand side participation, an effective protection scheme must take these fluctuations into account. Therefore, a fast controlled islanding approach which can act in real time and adapt to the currently existing generation and consumption conditions can offer a significant advantage. Here, a hybrid method is adopted. A slow coherency method is implemented, as described in Chow (1982) and in Koch et al. (2010), in order to provide an indication of the dynamic behavior of the generators during a disturbance. Subsequently, a method based on $k$-means clustering is introduced in order to identify in realtime the points where the network must be split after a large disturbance. The proposed approach acts fast and is able to adapt to the current generation and consumption conditions. It is, furthermore, modular, as different criteria can be incorporated for the identification of the islands.

In the following section, the $k$-means controlled islanding algorithm will be described and the main points of the proposed methodology will be analyzed.

\section{$2.2 k$-means Controlled Islanding}

The $k$-means algorithm is one of the widely known clustering methods in the field of machine learning. It was originally designed for partitioning $N$ observations into $K$ clusters, in which each observation belongs to the cluster with the nearest mean. An adaptation of the $k$-means algorithm for the controlled islanding problem is proposed in this section. The interested reader can refer to Bishop (2006) for the $k$-means algorithm formulation regarding data-points clustering.

The power system is modeled as a weighted, undirected graph $G=(V, E)$, where $V=\left\{v_{1}, \ldots, v_{n}\right\}$ is the set of vertices representing the $n$ power system nodes, $E=\left\{e_{i j}\right\}$ is the set of edges corresponding to the $m$ power lines and $W=\left\{w_{i j}\right\}$ is the set of weights assigned to the edges, i.e. $W: E \rightarrow \mathbf{R}$. From $E$ and $W$, a Weighted Adjacency Matrix of the graph can be built, $A=\left[a_{i j}\right]$, where

$$
a_{i j}=\left\{\begin{array}{rl}
w_{i j} & \text { if } e_{i j} \in E \\
0 & \text { otherwise }
\end{array} .\right.
$$

With the weights representing the distances between the nodes, we can influence how close or how far the nodes are from each other. The following criteria are used in the proposed approach in order to determine the weights:

(1) The groups of coherent generators according to the $\rho$ slowest modes,

(2) The rotor angle difference among the generators,
(3) The load-generation active power imbalance of the islands.

More details about the weight modification procedure will be given towards the end of this section. First, the $k$-means based algorithm for the partitioning of the system will be described.

From the Weighted Adjacency Matrix, the shortest path between any two buses, which we will refer to as "distance", is calculated (Cormen et al., 2001) and stored in the Distances Matrix $D=\left[d_{i j}\right]$.

The Distances Matrix is given as an input to the $k$-means algorithm. In the first step, a set of buses $\left\{\mu_{1}, \ldots, \mu_{k}\right\}$, where $k$ is the number of clusters, is selected. The $\left\{\mu_{k}\right\}$ correspond to the center of the clusters of the $k$-means algorithm, which we will refer to as centroids. The rest of the buses are assigned to one of the clusters according to the function:

$$
r_{n k}=\left\{\begin{array}{l}
1 \text { if } k=\operatorname{argmin}_{j} d_{n j} \\
0 \text { otherwise } .
\end{array}\right.
$$

In the second phase, within each cluster, the bus with the minimum distance from the rest of the buses is selected as the new centroid for this cluster:

$$
\mu_{k}=\operatorname{argmin}_{j} \sum_{i=1}^{M_{k}} d_{i j},
$$

where $d_{i j}$ is the distance between buses bus $s_{i}$ and $b u s_{j}$, with bus $_{i}$, bus $_{j} \in C_{k}$ ( $C_{k}$ being the cluster $)$, and $M_{k}$ being the total number of buses belonging to this cluster. As soon as the new centroids have been determined, the algorithm returns to the first step, where it assigns again all the buses to clusters. The algorithm converges when the set of centroids remains the same for two consecutive steps.

An overview of the approach is as follows: The controlled islanding algorithm is divided into two parts. The first one is executed off-line and the other runs in real time right after a disturbance occurs. For the off-line part, the weights are initialized to $w_{i j}=1, \forall i, j$, and subsequently get modified according to the slow coherency grouping. Generators belonging to the same coherent group are expected to exhibit similar behavior, when a disturbance occurs in the power system. For this reason, the distances between the generating nodes belonging to the same group are decreased. This modified matrix is taken as an input for the second part of the algorithm, which is executed as soon as a disturbance occurs. In the second part, the matrix is further modified so that the rotor-angle differences between the generators are taken into account. Generating nodes with similar rotor angles come closer together, while generator nodes with distant rotor angles get further apart. The modification of the Weighted Adjacency Matrix in this step is quite brute, since the splitting of the system into islands with similar relative angles plays an important role for the stability of the islands. The Distances Matrix is calculated at this point. Finally, the loadgeneration imbalance criterion is considered after this step, where the distances $d_{i j}$ between load nodes and nearby generators get decreased further. As nodes leading to a smaller loadgeneration imbalance "move" closer together, there is greater probability that the $k$-means algorithm groups these nodes together. In Fig. 1, an illustrative representation of the approach is given, while Fig. 2 presents the different steps of the algorithm in a flow chart. As soon as all criteria are considered, $k$-means is ready to take action. For a more detailed description of the different steps of the algorithm and the parameter settings, the interested reader can refer to Koch et al. (2010). 

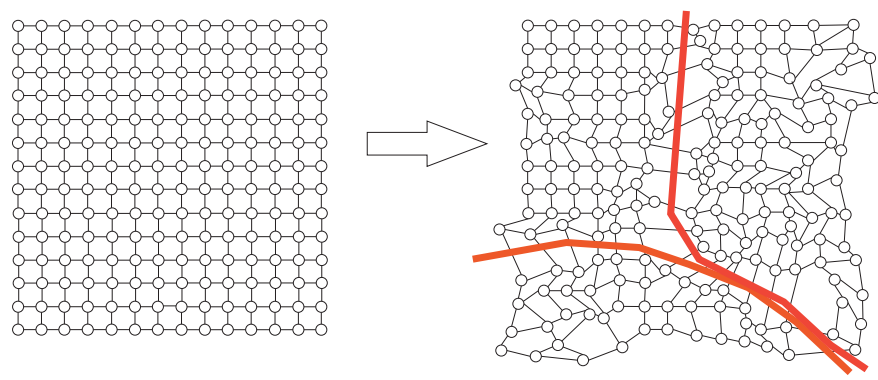

Fig. 1. Illustration of the modification of the Weighted Adjacency Matrix for $k$-means controlled islanding

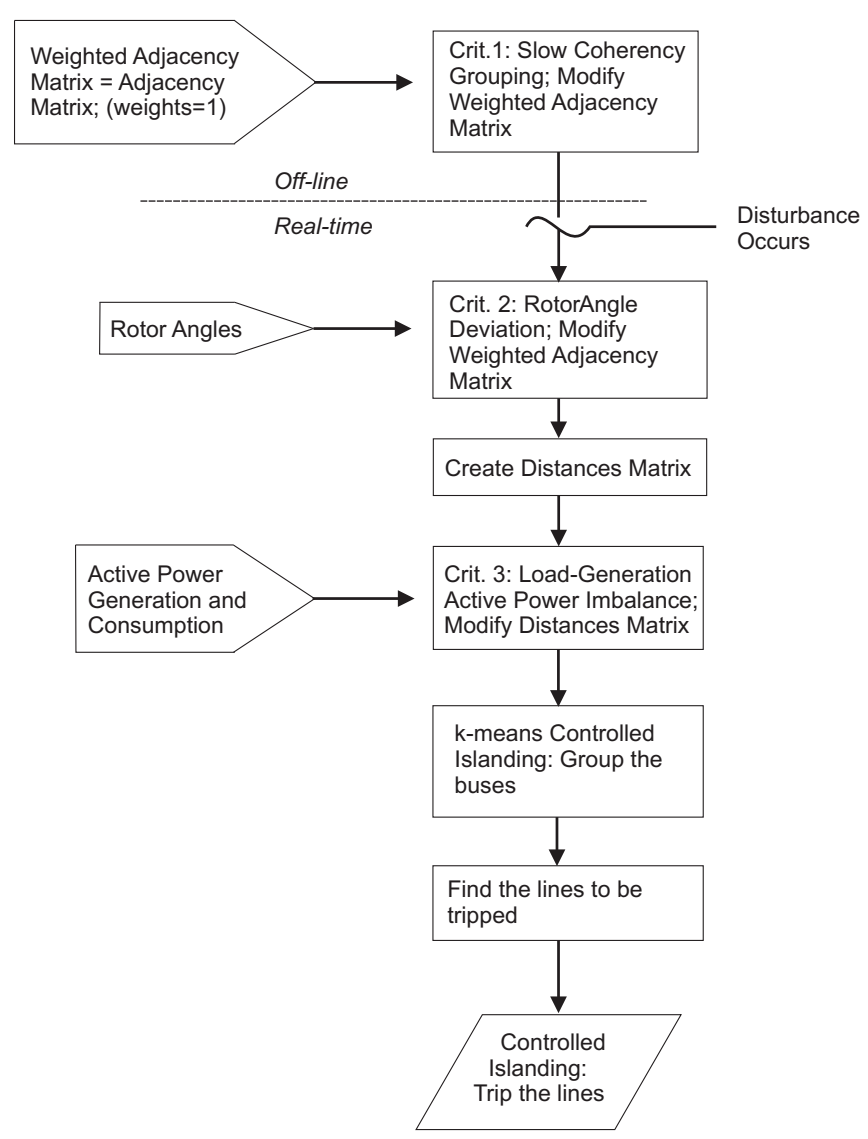

Fig. 2. Flow chart of the $k$-means controlled islanding algorithm

Controlled islanding based on the $k$-means approach exhibits certain advantages. The time needed for the execution of the real-time part of the algorithm is in the scale of milliseconds, and, therefore, it can act in real time. Furthermore, it can adapt to the currently existing conditions, thus it can potentially take into account intermittent generation and fluctuating loads. It is also modular allowing for the incorporation of more criteria by appropriately modifying the Weighted Adjacency Matrix or the Distances Matrix.

\subsection{Simulation example}

In order to illustrate the proposed method, time-domain simulations were conducted on the IEEE 118-bus system (IEEE, 1993). The dynamic data that were used in order to model the generators, the turbine governors and the Automatic Voltage Regulators can be found in Koch et al. (2010).
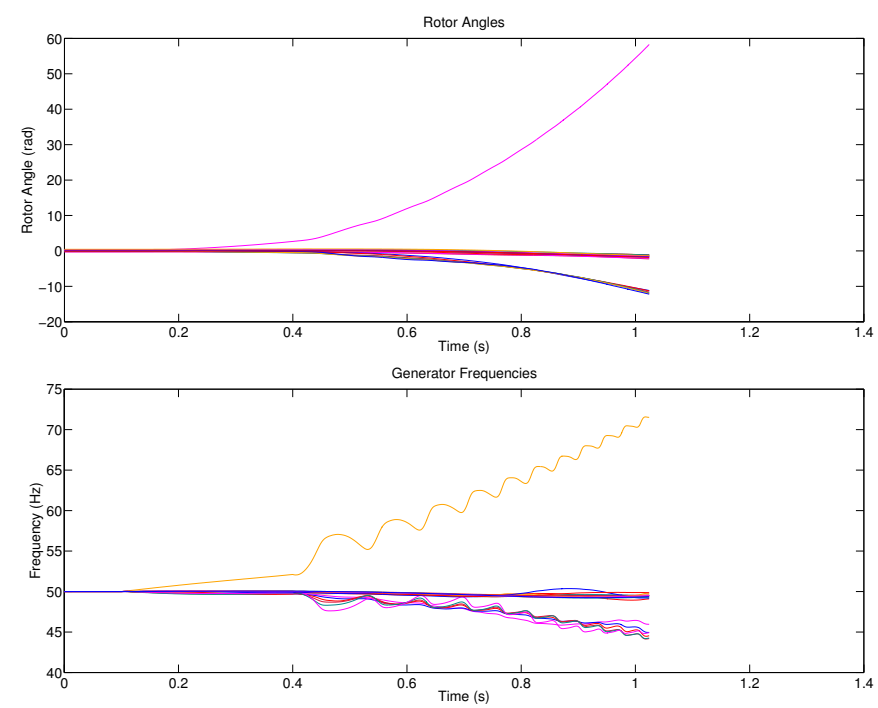

Fig. 3. Rotor angles and generator frequencies after the fault

A short-circuit on bus 65 is applied at $t=0.1 \mathrm{~s}$. After $300 \mathrm{~ms}$, the fault is cleared by tripping the lines $38-65$ and $65-68$. The effects of the disturbance are shown on Fig. 3. The angles of the generators on buses 46, 49, 54, 59, 61, 66 start to deviate from the rest of the generators, while the generator on bus 65 , about $500 \mathrm{~ms}$ after the disturbance, has already lost synchronism. Similar observations can be made on the Generator Frequencies graph. The generators that are losing their synchronism deviate from the nominal frequency. As a result, when crossing the frequency threshold of $47.5 \mathrm{~Hz}$, they have to be tripped. Such a fault would lead to a loss of generation of about 1369 MW which amounts to about $32 \%$ of the total generation in the system. The amount of load that should be shed would be similar, if not more.

The results after the proposed controlled islanding scheme are presented in Fig. 4 and Fig. 5. The algorithm requires about $242 \mathrm{~ms}$ in order to identify the three islands to be formed. To the first island, the algorithm assigns bus 65 . In order to form the second island, lines 34-43, 42-49, 46-47, 47-49, 4969, 64-65 and 65-66 are tripped. The lines are tripped at $t=$ $0.45 \mathrm{~s}$, namely $350 \mathrm{~ms}$ after the disturbance, accounting also for communication delays. Due to the controlled islanding scheme, bus 65 is isolated and will be tripped by the protection relays due to over-frequency. The rest of the generators will remain online, as none falls below $47.5 \mathrm{~Hz}$. In the other two islands, a total deficit of about $255.6 \mathrm{MW}$ is observed. This amounts to about $5.8 \%$ of the total generation.

The results show that a proper controlled islanding strategy can have a substantial effect on minimizing the amount of load shedding in the system. The method presented in this section has the potential to achieve this fast and effectively. Its positive effect can be further amplified by an efficient load shedding scheme, as the one that will be presented in the following section.

\section{CUSTOMER-LEVEL UNDER-FREQUENCY LOAD SHEDDING}

\subsection{Motivation}

One of the key aspects of power system security is the stability of the system frequency. In order to prevent the disconnection 


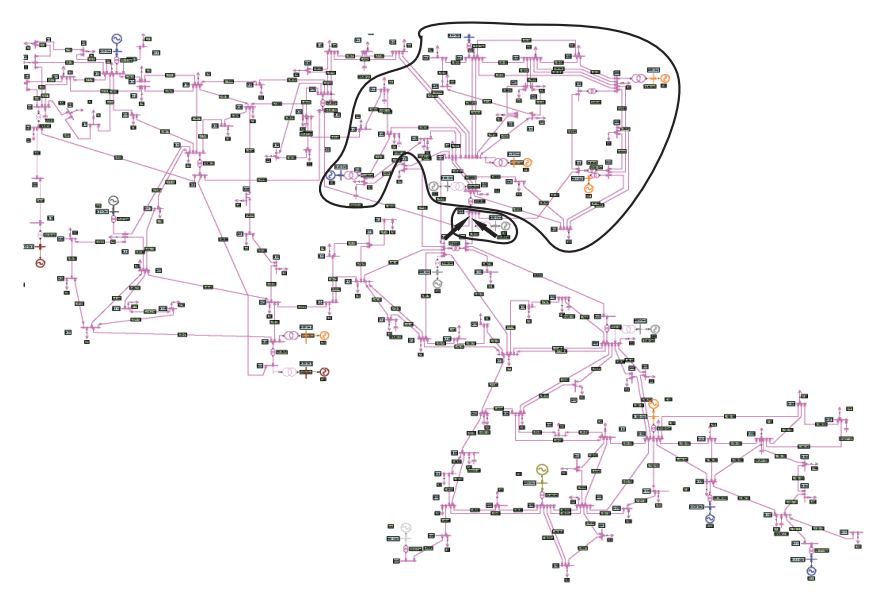

Fig. 4. Splitting of the power system after controlled islanding
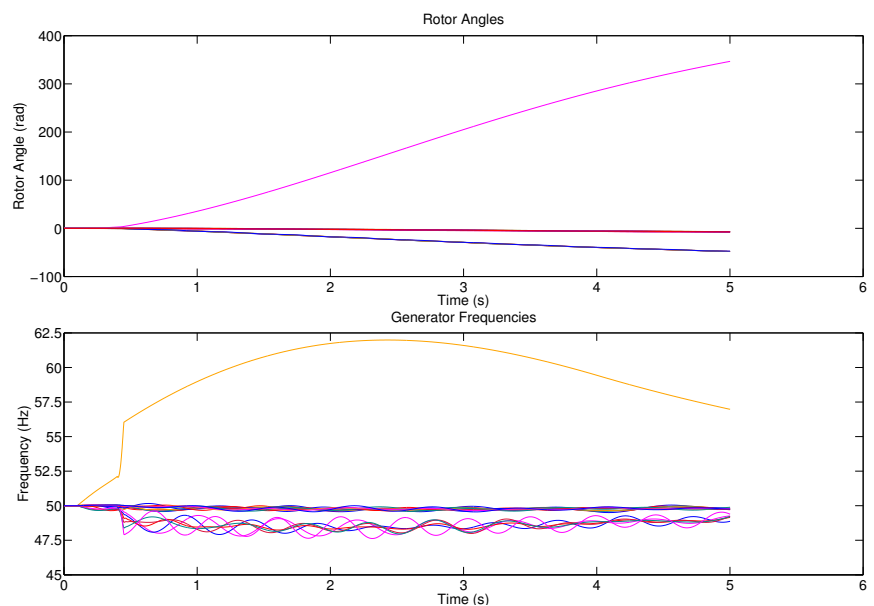

Fig. 5. Rotor angles and generator frequencies after the fault with Controlled Islanding

of power plants by their own protective equipment, a decay of the frequency below a certain threshold $(47.5 \mathrm{~Hz}$ in continental Europe where the nominal frequency is $50 \mathrm{~Hz}$ ) needs to be avoided. As standard frequency control measures such as primary control (ENTSO-E, 2004) may be insufficient to counteract large disturbances, under-frequency load shedding relays are in place in many countries as a last resort to stop a dangerous frequency decay. These are usually located on the lower-voltage side of the HV/MV transformers, or on subsequent $\mathrm{MV} / \mathrm{MV}$ or $\mathrm{MV} / \mathrm{LV}$ transformers in the distribution network. Thus, entire portions of the distribution network are de-energized by a relay tripping, which means that the consumers on these feeders are not supplied with any electricity. At the same time, all Distributed Generation (DG) units such as smaller wind farms, decentralized combined-heat-and-power (CHP) plants, or photovoltaic generation units on the shed feeders are lost. In the presence of high amounts of DG, the intended load shedding schemes can thus lead to significant involuntary generation shedding, which makes the system less and less effective as DG shares increase. One of the possible solution strategies is the migration of the shedding mechanism to the customer level, utilizing communication channels for assigning threshold frequencies to individual appliances. This would keep the distribution feeders energized and possibly installed DG units connected while minimizing the impact on the customer.

\subsection{Principle of operation}

Load Shedding Modeling Considered is a power system consisting of several transmission lines which interconnect a number of buses with central generators and distribution feeders. These feeders can be considered a model for "portions" of load that can be disconnected by a single frequency relay. Apart from the feeder subdivision, the load at each bus can be subdivided into load classes according to the associated functions that the load fulfills (such as cooking, washing, freezing, etc.). This is illustrated in Fig. 6.

Equations (3) - (5) show the nomenclature for the buses $n$, the load classes $i_{n}$, and the feeders $j_{n}$ at bus $n$ :

$$
\begin{aligned}
& \text { Bus: } n \in \mathfrak{N}=\{1, \ldots, N\}, \\
& \text { Load class at bus } n: i_{n} \in \mathfrak{L}_{n}=\left\{1, \ldots, N_{\mathrm{L}_{n}}\right\}, \\
& \text { Distr. feeder at bus } n: j_{n} \in \mathfrak{F}_{n}=\left\{1, \ldots, N_{\mathrm{F}_{n}}\right\} .
\end{aligned}
$$

For simplicity, the load classes are defined independently of the buses, so the number of load classes $N_{\mathrm{L}_{n}}$ is equal for all $n$ and they represent the same kind of load (e.g. residential cooling load). Note that this does not imply that all load classes have to be actually present at all buses.

The load at bus $n, P_{\mathrm{L}, n}$, can be modeled by commonly used aggregated load models for power system studies, e.g. as found in IEEE (1995). As the load shedding system will act independently of the load dynamics, the static load $P_{\mathrm{L} 0, n}$ at bus $n$ is considered for the computations. The same holds for the load classes and the feeder loads, as stated below.

For the subdivision of $P_{\mathrm{L} 0, n}$ into load classes $P_{\mathrm{L} 0, i_{n}}$, it is practical to define the ratios $r_{\mathrm{L}, i_{n}}$ between the individual load classes $i$ on bus $n$ and the total bus load. The load distribution onto the different feeders $j$ at bus $n$ is handled in the same way:

$$
\begin{aligned}
& r_{\mathrm{L}, i_{n}}=P_{\mathrm{L} 0, i_{n}} / P_{\mathrm{L} 0, n} \quad \\
& r_{\mathrm{F}, j_{n}}=P_{\mathrm{F} 0, j_{n}} / P_{\mathrm{F} 0, n} .
\end{aligned} .
$$

In order to render the notation more compact, these ratios can be written as ratio vectors for each bus $n$ in the system, the 1norm of which has to be equal to 1 by definition:

$$
\begin{aligned}
\mathbf{r}_{\mathrm{L}, n} & =\left[r_{\mathrm{L}, 1_{n}}, \ldots, r_{\mathrm{L}, N_{L_{n}}}\right]^{\mathrm{T}}, \\
\mathbf{r}_{\mathrm{F}, n} & =\left[r_{\mathrm{F}, 1_{n}}, \ldots, r_{\mathrm{L}, N_{F_{n}}}\right]^{\mathrm{T}} .
\end{aligned}
$$

The penetration ratio $r_{\mathrm{pen}, i_{n}} \in[0,1]$ is defined, which indicates the ratio of appliances in a class equipped with customer-level load shedding capability. This can also be gathered in the vector

$$
\mathbf{r}_{\text {pen }, n}=\left[r_{\text {pen }, 1_{n}}, \ldots, r_{\text {pen }, N_{L_{n}}}\right]^{\mathrm{T}}
$$

The load shedding system penetration is assumed to be uniform throughout the system, i.e. $\mathbf{r}_{\text {pen, } n}$ is equal for all $n$.

Distributed Generation model For the purposes of this work, Distributed Generation units are modeled as negative constantpower loads which are subdivided onto the distribution feeders introduced above. Further dynamics of the DG units is not considered, as they are assumed to be connected via inverters, and also distribution-level transformers which provide a certain amount of decoupling through their inductance. Note that the focus here is the interaction with under-frequency load shedding, and that autonomous disconnection of the DG through unit protection devices is not considered. The power that is injected by DG into bus $n$ is equal to $P_{\mathrm{DG}, n}$. In the same way as 


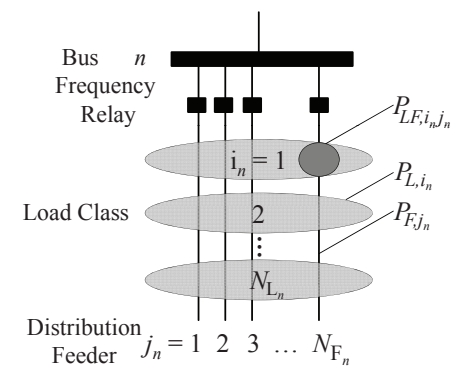

Fig. 6. Feeders and load classes at an arbitrary bus $n$

in equation (7), the distribution on the feeders emanating from bus $n$ is described by the ratio

$$
r_{\mathrm{DG}, j_{n}}=P_{\mathrm{DG}, j_{n}} / P_{\mathrm{DG}, n}
$$

or in vector notation for bus $n$ :

$$
\mathbf{r}_{\mathrm{DG}, n}=\left[r_{\mathrm{DG}, 1_{n}}, \ldots, r_{\mathrm{DG}, N_{F_{n}}}\right]^{\mathrm{T}} .
$$

Conventional load shedding The conventional load shedding system (C-UFLS) is composed of frequency relays (denoted by superscript fr) assumed to be present in every distribution feeder of the entire system. An arbitrary relay on feeder $j_{n}$ at bus $n$ is characterized by three parameters: the threshold frequency $f_{\mathrm{thr}, j_{n}}^{\mathrm{fr}}$, the detection time delay $\Delta t_{\text {detect, } j_{n}}^{\mathrm{fr}}$ and the tripping time delay $\Delta t_{\text {trip, } j_{n}}^{\mathrm{fr}}$. In case an under-frequency situation is detected, the shedding command is issued after $\Delta t_{\text {detect, } j_{n}}^{\mathrm{fr}}$ has passed and the measured frequency $f_{n}$ did not return above the threshold. Once the shedding command has been issued, the actual tripping is performed after $\Delta f_{\text {trip }, j_{n}}^{\mathrm{fr}}$.

We define now the remaining load at one feeder as the difference of the load before the shedding and the shed load:

$$
P_{\mathrm{F} 0, \text { rem }, j_{n}}=P_{\mathrm{F} 0, j_{n}}-P_{\mathrm{F} 0, \text { shed }, j_{n}} \quad .
$$

Corresponding to the load class and feeder ratios defined above, the shedding ratio of the feeder $j_{n}$ is defined as the relation of the currently shed load value to the value before the shedding:

$$
r_{\text {shed } j_{n}}^{\mathrm{fr}}=P_{\mathrm{L} 0, \text { shed }, j_{n}} / P_{\mathrm{L} 0, j_{n}} .
$$

As a single feeder is either shed or not shed, $r_{\text {shed }, j_{n}} \in\{0,1\}$ must hold. The following equations describe the logic of the shedding, taking into account the theoretical shedding (theo) which would be caused in the case of instantaneous shedding, the shedding if only detection time delay was considered (detect), and the true shedding including the tripping delay:

$$
\begin{aligned}
& r_{\text {shed }, j_{n}}^{\mathrm{fr}, \text { theo }}(t)=\left\{\begin{array}{ll}
0 & \text { for } f_{n}(t)>f_{\mathrm{tr}}^{\mathrm{fr}, j_{n}} \\
1 & \text { for } f_{n}(t) \leq f_{\mathrm{thr}, j_{n}}^{\mathrm{fr}}
\end{array},\right. \\
& r_{\text {shed }, j_{n}}^{\mathrm{fr} \text {,detect }}(t)=r_{\text {shed }, j_{n}}^{\text {fr,theo }}\left(\max _{\tilde{t} \in\left[t-\Delta t_{\text {detect, } \left.j_{n}, t\right]}^{\mathrm{fr}}, t\right.} f_{n}(\tilde{t})\right), \\
& r_{\text {shed }, j_{n}}^{\mathrm{fr}}(t)=r_{\text {shed }, j_{n}}^{\mathrm{fr}, \text { detect }}\left(t-\Delta t_{\text {trip }, j_{n}}\right) \quad .
\end{aligned}
$$

Having established the requirement that the load should not be reactivated automatically once the frequency returns above the threshold, the shedding ratio is restricted to be monotonically increasing, i.e. for the time instants $t_{1}$ and $t_{2}$ with $t_{1} \leq t_{2}$ holds

$$
r_{\text {shed }, j_{n}}^{\mathrm{fr}}\left(t_{1}\right) \leq r_{\text {shed }, j_{n}}^{\mathrm{fr}}\left(t_{2}\right)
$$

The main design parameters for the load shedding system are thus the frequency thresholds and the detection time delays of the frequency relays. The tripping time delay represents the latency of the frequency relay and can only be influenced by alteration of the tripping mechanism.
Customer-Level Load Shedding The shedding ratios of the customer-level load shedding (CL-UFLS, denoted by superscript $\mathrm{cl}$ ) are determined as follows: based on the ramp-wise load shedding emulation outlined in Koch et al. (2010), two boundaries for frequency thresholds have to be given to each load class. The actual threshold of each appliance between these boundaries is then determined on the household level. In an analogous way to the conventional load shedding, we define the shed and remaining load caused by one load class $i_{n}$ at bus $n$, as well as the shedding ratio:

$$
\begin{aligned}
P_{\mathrm{L} 0, \text { rem }, i_{n}} & =P_{\mathrm{L} 0, i_{n}}-P_{\mathrm{L} 0, \text { shed }, i_{n}}, \\
r_{\text {shed }, i_{n}}^{\mathrm{cl}} & =P_{\mathrm{L} 0, \text { shed }, i_{n}} / P_{\mathrm{L} 0, i_{n}} .
\end{aligned}
$$

As the load shedding is performed ramp-wise, $r_{\text {shed }, j_{n}} \in[0,1]$ holds in contrast to the conventional load shedding. The following equations describe the logic of the shedding, taking into account the theoretical shedding (theo) which would be caused in the case of instantaneous shedding, the shedding if only detection time delay was considered (detect), and the true shedding including the tripping delay:

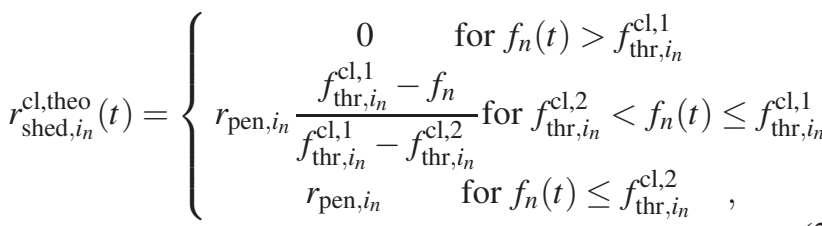

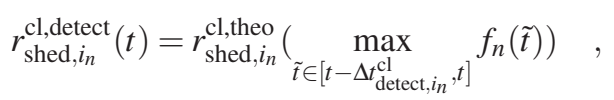

$$
\begin{aligned}
& r_{\text {shed }, i_{n}}^{\mathrm{cl}}(t)=r_{\text {shed }, i_{n}}^{\mathrm{cl}, \mathrm{det}}\left(t-\Delta t_{\text {trip }, i_{n}}^{\mathrm{cl}}\right) \quad .
\end{aligned}
$$

Same as in the case of conventional load shedding, the shedding ratio is restricted to be monotonically increasing, i.e. for the time instants $t_{1}$ and $t_{2}$ with $t_{1} \leq t_{2}$ holds

$$
r_{\text {shed, } i_{n}}^{\mathrm{cl}}\left(t_{1}\right) \leq r_{\text {shed, }, i_{n}}^{\mathrm{cl}}\left(t_{2}\right)
$$

Remaining Load at Bus n Any combination of the previously modeled C-UFLS system and the newly introduced CL-UFLS system has to take into account the fact that the same load can only be tripped once. Thus, the effects of both load shedding systems have to be concatenated. This requires one further assumption, namely the distribution of one individual load class at one bus onto the feeders at this bus. It is assumed here that a certain load class $P_{\mathrm{L}, i_{n}}$ at bus $n$ is equally distributed onto the $N_{\mathrm{F}_{n}}$ feeders at this bus, i.e.

$$
P_{\mathrm{LF}, i_{n} j_{n}}=P_{\mathrm{L}, i_{n}} / N_{\mathrm{F}_{n}} \quad \forall i_{n} \in \mathfrak{L}_{n}, j_{n} \in \mathfrak{F}_{n} \quad .
$$

Under this requirement, the concatenation of customer-level and conventional load shedding is performed in the following way: the remaining load at the bus due to a certain customerlevel load shedding ratios $\mathbf{r}_{\mathrm{L}, n}$ is calculated, which is then subject to a further reduction by the conventional load shedding ratio $\mathbf{r}_{\mathrm{F}, n}$. The remaining load after the customer-level load shedding is described by

$$
P_{\mathrm{L} 0, \text { rem }, n}^{\mathrm{cl}}=\mathbf{r}_{\mathrm{L}, n}^{\mathrm{T}}\left[\mathbf{1}-\mathbf{r}_{\mathrm{shed}, n}^{\mathrm{cl}}\right] P_{\mathrm{L} 0, n}
$$

The effect of the conventional load shedding is independent of the class-dependent load reduction because of the requirement from equation (25). Thus, it can be assumed to act on the aggregated remaining load after the customer-level load shedding:

$$
P_{\mathrm{L} 0, \mathrm{rem}, n}^{\mathrm{fr}}=\mathbf{r}_{\mathrm{F}, n}^{\mathrm{T}}\left[\mathbf{1}-\mathbf{r}_{\mathrm{shed}, n}^{\mathrm{fr}}\right] P_{\mathrm{L} 0, \mathrm{rem}, n}^{\mathrm{cl}} .
$$


Inserting equation (26) into (27), the remaining load at bus $n$ is equal to:

$$
P_{\mathrm{L} 0, \mathrm{rem}, n}=\mathbf{r}_{\mathrm{F}, n}^{\mathrm{T}}\left[\mathbf{1}-\mathbf{r}_{\mathrm{shed}, n}^{\mathrm{fr}}\right] \cdot \mathbf{r}_{\mathrm{L}, n}^{\mathrm{T}}\left[\mathbf{1}-\mathbf{r}_{\mathrm{shed}, n}^{\mathrm{cl}}\right] P_{\mathrm{L} 0, n} \quad .
$$

\subsection{Simulation example}

As an example, the effect of a large under-frequency disturbance in the IEEE 118-bus system (IEEE, 1993) is simulated. The setup is largely based on Koch et al. (2010), with the following differences: the disturbance scenario considered here is a simultaneous outage of the generators at bus 80 and 89 , which are relatively close to each other; furthermore, a $50 \%$ DG power penetration is considered, Poisson-distributed in 0.2 MW portions on the system feeders. The power generation of the central generators is reduced proportionally. This can be justified if the considered DG is only temporarily available generation such as photovoltaic generation around noon, which is not reliable enough to make a substantial impact on unit commitment.

Three simulated scenarios are shown: a) no DG penetration (and, consequently, no DG loss) and conventional load shedding, b) $50 \%$ DG and conventional load shedding, c) $50 \%$ DG and 50\% penetration of customer level load shedding with adaptation of the conventional load shedding thresholds according to Koch et al. (2010). Fig. 7 shows the simulated evolution of the generator frequencies for the three cases. It can be seen that in case a) (upper plot) the load shedding is sufficient to bring the system frequency back to the vicinity of its nominal value. In Case b) (middle plot), the amount of shed load is the same as in a) because the same frequency relays are triggered. However, the frequency is not brought back to its nominal value due to simultaneous DG shedding. This effect is alleviated in Case c) (lower plot) by the customer-level load shedding that prevents the activation of the conventional load shedding. The lost load in the latter case is slightly larger than in the first two cases due the load shedding system parameterization. However, the disconnected load consists entirely of low-priority load, which does not cause any disruption for the user in the case of a short interruption.

The involuntary DG shedding does not lead to additional load shedding in the present simulation. This is due to the fact that in case a) more load is shed than necessary for a frequency stabilization, which leads to the frequency rise back to the nominal value observed in the upper plot in Fig. 7. Thus, the difference between case a) and b) consists of a difference in steady-state frequency. In the case of a larger initial disturbance exactly matching the first conventional load shedding stage, the load shedding in case b) would be larger corresponding to the amount of DG lost.

\section{PREVENTION OF GRID OVERLOADING THROUGH PHEVS}

\subsection{Motivation}

Plug In Hybrid Electric Vehicles (PHEVs) are cars which use electricity for propulsion while utilizing gasoline only as an auxiliary power source (Williams and Kurani, 2007).

Wide scale PHEV adoption is considered as an attractive option to decarbonize private transport when recharging the vehicles with energy generated by fluctuating renewable energy sources.
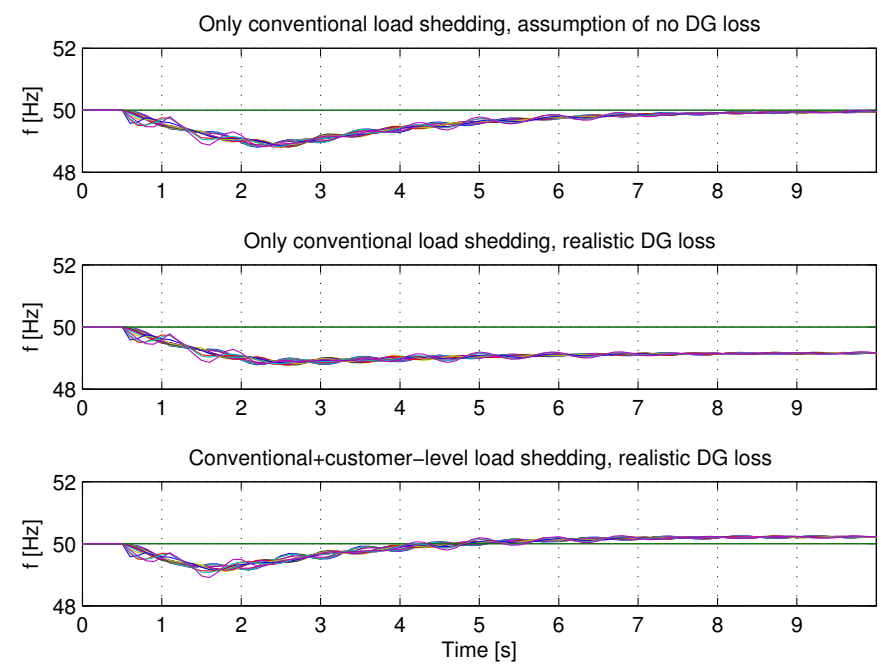

Fig. 7. Load shedding results

As the PHEVs penetrate the existent electricity infrastructure, additional load is imposed, the spatial and temporal distribution of which is not yet anticipated. This introduces uncertainty in planning stages and in the operation of the power system (Galus et al., 2010).

The clustering of vehicles in one supply area can cause overloading of transformers and lines or can induce overly deep voltage sags (Pecas Lopes et al., 2009). Therefore, effective demand management schemes for PHEVs are developed (Galus et al., 2011) which can avoid such negative impacts while optimally allocating scarce power to a large number of cars according to their individual transportation demands.

\subsection{Principle of operation}

The demand management approach presented in the following is based on agent and on utility theory. The approach assigns a utility function to each managed vehicle. The function attributes a value which is expressed in monetary terms to the individual battery energy level of the PHEV. The utility function, the rationale of which is explained below, is formulated as

$$
\begin{gathered}
u_{k_{n}}\left(q_{k_{n}}\left(T, \theta_{k_{n}}(T) \mid \Theta_{n}(T)\right), S O C_{k_{n}}(T), S O C_{k_{n}}^{\min }, \pi(T), \theta_{k_{n}}(T)\right)= \\
\theta_{k_{n}}(T) \alpha_{k_{n}} C_{k_{n}}^{\mathrm{B}}\left(S O C_{k_{n}}(T)-S O C_{k_{n}}^{\min }+q_{k_{n}}\left(T, \theta_{k_{n}}(T) \mid \Theta_{n}(T)\right)\right) \\
-\theta_{k_{n}}(T) \beta_{k_{n}} C_{k_{n}}^{\mathrm{B}}\left(S O C_{k_{n}}(T)-S O C_{k_{n}}^{\min }+q_{k_{n}}\left(T, \theta_{k_{n}}(T) \mid \Theta_{n}(T)\right)\right)^{2} \\
-\pi\left(T, \Theta_{n}(T)\right) C_{k_{n}}^{\mathrm{B}} q_{k_{n}}\left(T, \theta_{k_{n}}(T) \mid \Theta_{n}(T)\right) \\
\forall k_{n} \in \mathscr{N}_{n}(T)=\left\{1 \ldots N_{n}^{\mathrm{PHEV}}(T)\right\}, \\
\forall n \in \mathscr{N}=\{1 \ldots N\} .
\end{gathered}
$$

The utility is dependent on the actual state of charge $S O C_{k_{n}}(T)$ in time interval $T$ and on the allowed minimal state of charge $S O C_{k_{n}}^{\min }$ of vehicle $k_{n} \in \mathscr{V}_{n}(T)$ at network node $n \in \mathscr{N}$. The parameter $C_{k_{n}}^{\mathrm{B}}$ denotes the vehicle's battery capacity and $\theta_{k_{n}}(T)$ the individual vehicle type.

The first two summands of the utility function, when not multiplied by $\theta_{k_{n}}(T)$, are called the benefit function

$$
B_{k_{n}}\left(q_{k_{n}}\left(T, \theta_{k_{n}}(T) \mid \Theta_{n}(T)\right), S O C_{k_{n}}(T), S O C_{k_{n}}^{\min }\right) .
$$

The function is monotonically nondecreasing in SOC and continuously differentiable. The benefit function incorporates the tuning parameters $\alpha_{k_{n}}$ and $\beta_{k_{n}}$ which are expressed in monetary 


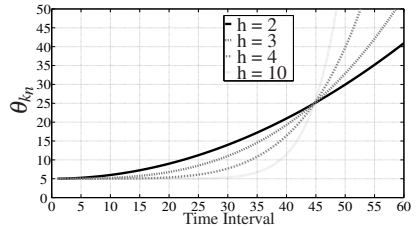

(a) Plot of vehicles' personal type

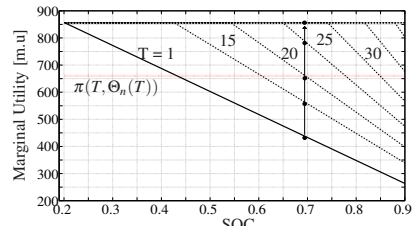

(b) Plot of the vehicles' marginal utility over time
Fig. 8. Functionality of PHEV management scheme showing type and marginal utility evolution over time

terms. These parameters determine the recharging behavior related to an exogenously given, or, when congestion occurs, an endogenously determined, energy price signal. Put in another way, the parameters determine the sensitivity for acquiring the infinitesimal energy amount $q_{k_{n}}\left(T, \theta_{k_{n}}(T) \mid \Theta_{n}(T)\right)$ in time step $T$ in relation to a price-based control signal. The parameter $\alpha_{k_{n}}$ is set to the gasoline price weighted by the combustion engine efficiency. This ensures that recharging does not occur for price signals higher than this value.

The vehicle type $\theta_{k_{n}}(T)$ in the utility function is a weighting factor increasing the sensitivity to acquire energy. It is given through a power law which depends on the actual $S O C$, the desired $S O C$ at departure, the potentially chargeable energy in $T$ and the time left to departure (Galus and Andersson, 2008; Galus et al., 2011). Hence, it expresses the urgency of the vehicle to acquire energy before departure. The parameter is depicted in Fig. 8(a) for a vehicle with an SOC of 30\%, a desired SOC of $90 \%$, an anticipated departure at time step 60 and for different exponents $h$. Obviously, the parameter increases with less parking time, i.e. potential recharging time. It is noted that the vehicle is not recharged here for simplicity. The parameter is also called personal energy valuation (Galus and Andersson, 2008). Finally, the acquired energy amount is dependent on the own type $\theta_{k_{n}}(T)$, on the set of types $\Theta_{n}(T)$ of all other present PHEVs at the particular node and on the current energy price signal $\pi\left(T, \Theta_{n}(T)\right)$ which is included in the utility function.

The functionality of the demand management scheme is illustrated in Fig. 8(b). Here, the horizontal line denotes the price signal level which is kept constant in the following argumentation for simplicity. The black lines depict the marginal weighted benefit, i.e. $\mathrm{d} \theta_{k_{n}}(T) B_{k_{n}}(\cdot) / \mathrm{d} q_{k_{n}}(\cdot)$ for a vehicle which is anticipated to depart at $T=60$ and which features a $S O C$ of $70 \%$. During $T=1$, the marginal weighted benefit is obviously smaller than $\pi\left(T, \Theta_{n}(T)\right)$ and therefore the vehicle will not recharge as it would lose utility in doing so. However, as the vehicle does not acquire any energy over several time intervals, the vehicle type, i.e. personal energy valuation, increases, and therefore the marginal weighted benefit grows until it is larger than $\pi\left(T, \Theta_{n}(T)\right)$. If the vehicle should still not acquire energy, as shown here, the benefit will grow further until it reaches the maximum value given by $\alpha_{k_{n}}$. The sensitivity of acquiring energy, i.e. the slope, increases with rising values of the vehicle type. Tuning the parameters $\alpha_{k_{n}}$ and $\beta_{k_{n}}$ allows for the integration with transportation models (Galus et al., 2011) or/and for favorable recharging behavior over time, e.g. valley filling.

The utility-based model can be used to avoid negative network impacts through wide scale PHEV adoption by introducing a PHEV management platform. This platform, from here on called PHEV Manager, is envisioned to be present at each power system node and to aggregate and manage the demand

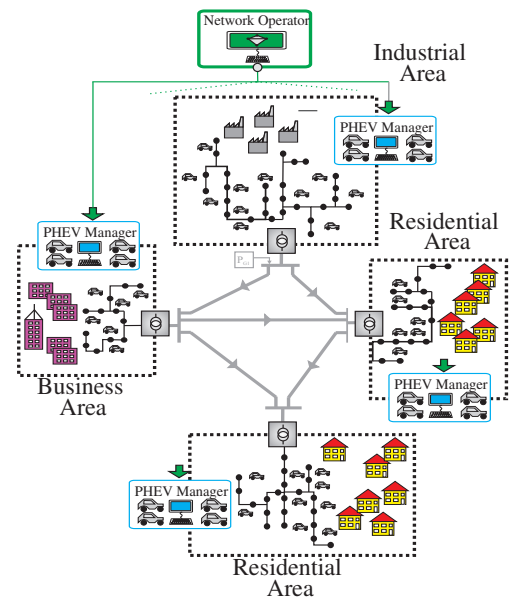

Fig. 9. PHEV number over time at all system nodes

of large numbers of vehicles, e.g. at medium voltage network nodes which typically feed larger areas. In case of line or transformer overload, the PHEV Manager sheds PHEV load while allocating the available power optimally to the connected vehicles. The scheme endogenously determines control price signals $\pi\left(T, \Theta_{n}(T)\right)$, which lead to a corresponding energy demand decrease of the vehicles. In order to determine the price signals, each PHEV Manager performs an optimization which maximizes

$$
\underset{k_{n} \in \mathscr{Y}_{n}(T)}{\sum u_{k_{n}}\left(q_{k_{n}}\left(T, \theta_{k_{n}}(T) \mid \Theta_{n}(T)\right), S O C_{k_{n}}(T), S O C_{k_{n}}^{\min }, \pi(T), \theta_{k_{n}}(T)\right)}
$$

subject to

$$
\begin{aligned}
& 0 \leq q_{k_{n}}\left(T, \theta_{k_{n}}(T) \mid \Theta_{n}(T)\right) \leq \bar{q}_{k_{n}}(T) \quad(a) \\
& \underline{S O C}_{k_{n}} \leq \quad \operatorname{SOC}_{k_{n}}(T) \leq 1 \quad(b) \\
& \left.0 \leq \sum_{k_{n} \in \mathscr{V} n} q_{k_{n}}(T) \theta_{k_{n}}(T) \mid \Theta_{n}(T)\right) \frac{C_{k_{n}}^{B}}{\tau} \leq P_{n}^{\mathrm{PHEV}}(T)(c) \\
& \forall k_{n} \in \mathscr{V}_{n}(T), \forall n \in \mathscr{N}
\end{aligned}
$$

where $\bar{q}_{k_{n}}(T)$ denotes the maximal attainable energy for one vehicle during one time interval. It is expressed in terms of SOC. The variable $T$ denotes the time interval of length $\tau$, which is chosen to $\tau=900 \mathrm{~s}$. Constraint (31b) bounds the individual SOCs and (31c) determines that the sum of the individual power consumptions does not exceed the maximal power $P_{n}^{\text {PHEV }}(T)$ which can be provided to the PHEV s at node $n$ (Galus and Andersson, 2008; Galus et al., 2011).

\subsection{Simulation example}

To illustrate the demand management scheme, a four node test network, shown in Fig. 9, is utilized with PHEV Managers present at each network node. The managers are assumed to be controlled by the network operator, e.g. the distribution system operator. The network is envisioned to interconnect several larger urban areas, therefore the lines are modeled with the well known DC-approximation (Wood and Wollenberg, 1996). Each line has a thermal limit, e.g. a maximal current which it can carry. The transformers in the system incorporate a maximal power rating of $50 \mathrm{MW}$ and an efficiency $\eta$ of $98 \%$. The system without PHEVs is secure but heavily loaded. Different area specific load curves are shown in Fig. 10, which have been used to realistically represent the network loading. 


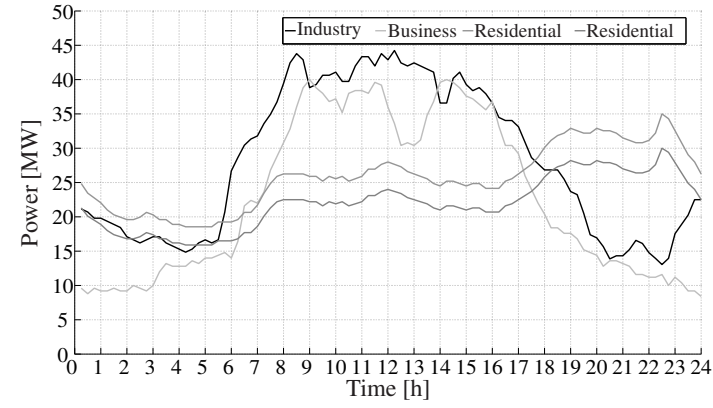

Fig. 10. Load curves for the modeled urban areas

The daily behavior of the PHEVs including their driving patterns have been simulated through the model elaborated in Galus et al. (2011). The PHEVs partly switch areas during the day in order to commute to work and back. Their behavioral patterns rest upon a transportation network which is mapped to the electricity network. The vehicle energy consumption when driving is determined through the model discussed in Galus and Andersson (2009).

The electricity system is simulated using the optimal power flow approach (OPF). As the system does not feature generators, it is assumed that the slack node, set as bus number one, provides energy at a constant, exogenous price $\pi(T)$. This price $\pi(T)$ forms the basis for the endogenous control price signal determination through the optimization of the PHEV Manager. The OPF is computed by minimizing

$\sum_{n=1}^{N}\left(\pi(T) L_{n}(T)+\xi\left(P_{n}^{\text {PHEV }}(T)-P_{n}^{\text {PHEV,act }}(T)\right)\right) \tau$,

subject to

$$
\begin{array}{ll}
\mathbf{L}_{n}(T)=\mathbf{L}_{n}^{\text {base }}(T)+\mathbf{P}_{n}^{\text {PHEV,act }}(T) & \forall n \in \mathscr{N}, \\
\mathbf{L}_{n}(T)-\eta_{n} \mathbf{P}_{n}(T)=\mathbf{0} & \forall n \in \mathscr{N}, \\
\mathbf{G}\left(\mathbf{P}_{n}(T)\right)=\mathbf{0} & \forall n \in \mathscr{N},
\end{array}
$$

and

$$
\begin{array}{llr}
\underline{\mathbf{P}}_{n} \leq \mathbf{P}_{n}(T) & \leq \overline{\mathbf{P}}_{n} & \forall n \in \mathscr{N} \\
\underline{\mathbf{P}}_{l} \leq \mathbf{P}_{l}(T) & \leq \overline{\mathbf{P}}_{l} & \forall l \in \mathscr{L} \\
\mathbf{0} \leq \mathbf{P}_{n}^{\text {PHEV,act }}(T) & \leq \mathbf{P}_{n}^{\text {PHEV }}(T) & \forall n \in \mathscr{N} \\
\phi_{n}=0 & & n=1
\end{array},
$$

The objective function, given by (32), incorporates the cost of the consumed energy in the first summand. The PHEV load imposed by the PHEV managers is considered as a flexible load in contrary to the base load of the system. In case of system overloading, the flexible PHEV load can be curtailed. The second term in the objective function penalizes the deviation between the energy $P_{n}^{\text {PHEV,act }}(T) \cdot \tau$ actually provided to the PHEVs and the desired energy $P_{n}^{\mathrm{PHEV}}(T) \cdot \tau$ of the respective PHEV Manager. The parameter $\xi^{n}$ is a penalization factor set to a high value to avoid excessive PHEV shedding.

Equation (33a) expresses the total load at each node, (33b) ensures that the power fed by the transformers, the efficiency of which is given by $\eta_{n}$, has to correspond to the load at the node. Equation (33c) ensures that Kirchhoff's law is obeyed.

The constraint (34a) determines that the power input to an area, i.e. the power which can be fed through the transformer at each node, is limited. The electric line limits are expressed through (34b) while (34c) describes the potential for curtailing the PHEV load at each node. Finally, (34d) sets the reference angle to compute the power flow.

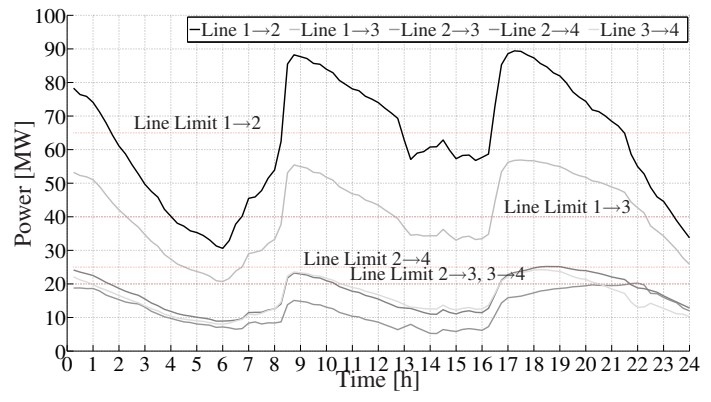

(a) Plot of line flows without shedding of PHEVs

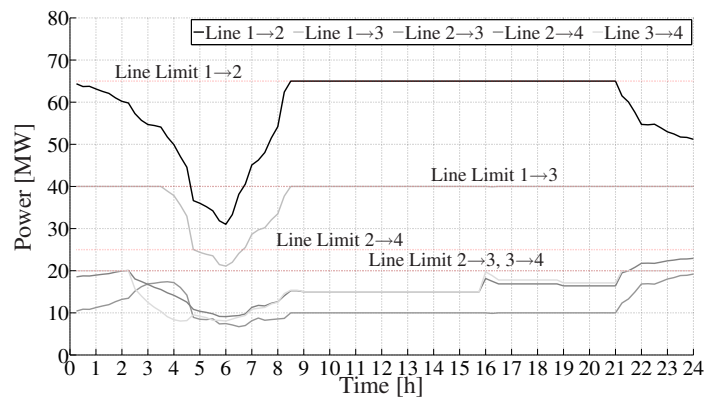

(b) Plot of line flows with shedding of PHEVs

Fig. 11. Optimal power flow shedding PHEV load to incorporate physical transformer and line power limitations

Figure 11 shows the power flows on the lines computed through the OPF. Figure 11(a) displays the case where the PHEV load is not curtailed. Obviously, several line limits are heavily violated and the system is massively overloaded. Most of the line loadings largely exceed their respective thermal limits. No transformer is overloaded due to the setup of the PHEV Manager optimization (30) - (31). Taking advantage of the flexible PHEV load, the PHEV demand is distributed over longer time intervals in order to relieve the heavily loaded lines as illustrated in Fig. 11(b). Here, the PHEV load is curtailed at times where the network overloads. Therefore no line limits are violated. It can be seen that the large load peaks are flattened and the PHEV load is shifted to later times.

Figure 12 depicts the evolution of the control price signals which are computed via the PHEV Manager optimization scheme. In the case of Fig. 12(a) the control price signals stay either at the constant exogenously given level or show peaks which are due to congestion of the transformers at the nodes. The line limits are not considered in this case. In Fig. 12(b) the control price signals are plotted when considering the thermal line limits, and curtailing and redistributing the PHEV. Obviously, the price signals are much higher than in Fig. 12(a) because more PHEVs need to be shed during certain time intervals in order to relieve lines. The control price signals are more often endogenously determined by the PHEV Manager optimization, avoiding excessive PHEV charging. However, the available power at the nodes is optimally distributed between the connected PHEVs taking into account their individual, temporal transportation demands.

\section{CONCLUSION AND OUTLOOK}

Significant challenges for power system security are associated with the structural transition towards a sustainable and highly distributed energy system which incorporates electro-mobility. New control methods, the penetration of which is largely linked 


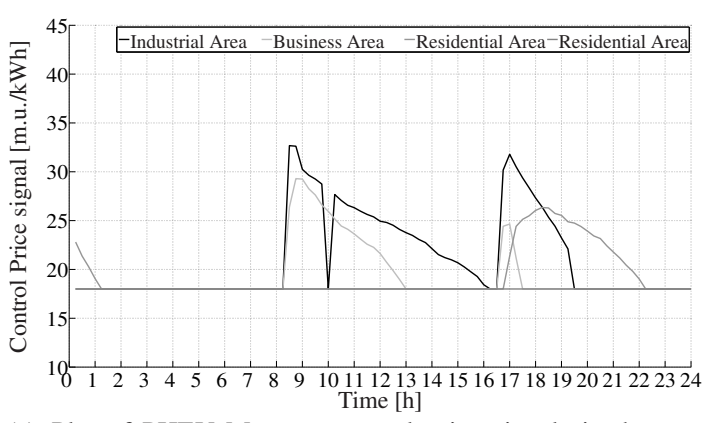

(a) Plot of PHEV Manager control price signals in the case where line constraints are neglected

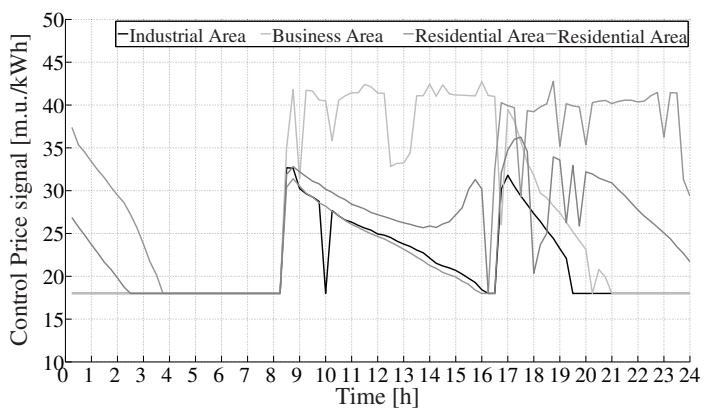

(b) Plot of PHEV Manager control price signals in the case where line constraints are incorporated

Fig. 12. Control price signals of PHEV Managers

to the penetration of advanced information and communication technologies, will need to take into account the heterogeneity of the system and different time scales on which effects need to be controlled for ensuring system stability.

This paper discusses three control methodologies effective on different time scales, exemplifying the challenges for future integration of control methods into power systems. Clearly, $k$ means based controlled islanding is active on much shorter time frames than the PHEV scheduling technique. However, in order to ensure system security, the three proposed methodologies need to be integrated into a consistent framework of emergency control strategies that interacts in a predictable way. Approaches towards such a framework are starting to be developed as in e.g. Ulbig et al. (2011).

\section{REFERENCES}

Belmans, R. (2007). Liberalized markets and technical boundary conditions. The Belgian energy policy towards 2030 within a European framework. URL http: / / www. ce2030. be.

Bialek, J. (2007). Why has it happened again? Comparison between the UCTE blackout in 2006 and the blackouts of 2003. In Proceedings of IEEE PowerTech. Lausanne, Switzerland.

Bishop, C.M. (2006). Pattern Recognition and Machine Learning. New York: Springer-Verlag.

Bollen, M. and Samuelsson, O. (2009). Controlled island operation of part of the $50-\mathrm{kV}$ grid in Southern Sweden. In Proceedings of IEEE PowerTech. Bucharest, Romania.

Chow, J.H. (1982). Time-Scale Modeling of Dynamic Networks with Applications to Power Systems. New York: SpringerVerlag.

Cormen, T.H., Leiserson, C.E., Rivest, R.L., and Stein, C. (2001). Introduction to Algorithms. Cambridge, Massachussets: MIT Press.
ENTSO-E (2004). Continental Europe (CE) Operation Handbook. URL http: / / www . ent soe.eu.

Galus, M.D. and Andersson, G. (2008). Demand management for grid connected plug-in hybrid electric vehicles (PHEVs). In Proceedings of IEEE Energy 2030. Atlanta, GA, USA.

Galus, M.D. and Andersson, G. (2009). Power system considerations of plug-in hybrid electric vehicles based on a multi energy carrier model. In Proceedings of IEEE Power and Energy Society (PES) General Meeting. Calgary, Canada.

Galus, M.D., Waraich, R.A., Noembrini, F., Steurs, K., Boulouchos, K., Axhausen, K.W., and Andersson, G. (2011). Integrating power systems, transport systems and vehicle technology for load, behavioral and environmental analysis of electric mobility. Submitted to: IEEE Transactions on Smart Grids.

Galus, M.D., Zima, M., and Andersson, G. (2010). On integration of PHEVs into existing power system structures. Energy Policy, (38), 6736-67.

He, Y., Jenkins, N., Wu, J., and Eltayeb, M. (2010). ICT infrastructure for smart distribution networks. In IEEE International Symposium on Power Line Communications and Its Applications (ISPLC).

IEEE (1993). Power Systems Test Case Archive, College of Engineering, University of Washington.

IEEE (1995). Standard load models for power flow and dynamic performance simulation. IEEE Transactions on Power Systems, 10(3), 1302 -1313. doi:10.1109/59.466523.

Koch, S., Chatzivasileiadis, S., Vrakopoulou, M., and Andersson, G. (2010). Mitigation of cascading failures by realtime controlled islanding and graceful load shedding. In IREP Symposium 2010 - Bulk Power System Dynamics and Control - VIII. Buzios, RJ, Brazil.

Li, H., Rosenwald, G., Jung, J., and Liu, C. (2005). Strategic power infrastructure defense. Proceedings of the IEEE, 93(5), $918-933$.

Pecas Lopes, J.A., Soares, F.J., and Rocha Almeida, P.M. (2009). Identifying management procedures to deal with connection of electric vehicles in the grid. In Proceedings of IEEE PowerTech. Bucharest, Romania.

Sena, C., Taranto, G.N., and Giusto, A. (2010). An investigation of controlled power system separation of the Uruguayan network. In IREP Symposium 2010 - Bulk Power System Dynamics and Control - VIII. Buzios, RJ, Brazil.

Sun, K., Zheng, D.Z., and Lu, Q. (2005). A simulation study of OBDD-based proper splitting strategies for power systems under consideration of transient stability. IEEE Transactions on Power Systems, 20(1), 389 - 399.

UCTE (2007). Final Report - System Disturbance on 4 November 2006. URL https : / / www . ent soe . eu.

Ulbig, A., Arnold, M., Chatzivasileiadis, S., and Andersson, G. (2011). Framework for multiple time-scale MPC application in power systems. In IFAC World Congress 2011. Milano, Italy.

Williams, B.D. and Kurani, K.S. (2007). Commercializing light-duty plug-in/plug-out hydrogen-fuel-cell vehicles: "mobile electricity" technologies and opportunities. Journal of Power Sources, 166(2), 549-566.

Wood, J.W. and Wollenberg, F.B. (1996). Power generation, operation and control. Wiley-Interscience, 2 nd edition.

You, H., Vittal, V., and Wang, X. (2004). Slow coherency-based islanding. IEEE Transactions on Power Systems, 19(1), 483491. 